\title{
Estudio De La Motivación Y Satisfacción Del Profesorado Para El Desempeño Profesional En Benguela (Angola)
}

\author{
Lúcia Fernanda Dassala Chipuca \\ Subdirectora Pedagógica Escola Ensino Secundário Novembro de Benguela
}

Inmaculada Aznar Díaz

Profesora Titular de Universidad. Universidad de Granada.

\section{Antonio-Manuel Rodríguez-García}

PDI. Universidad de Granada.

\begin{abstract}
There are a few researches about motivation and job satisfaction, but we found some studies about teacher motivation and their satisfaction in their workplace. In Angola's context, this topic emerges as a new field to research inside the school organization and management area, understanding this as body of knowledge and actions among people who converge on shared values and practices which are manifested through work. This papper deals with the study of teacher's motivation and satisfaction for their professional performance in Benguela (Angola). Through it, we try to describe how the motivation and satisfaction can influence on the professional development of teachers who work in Secondary Education in Benguela. It is performed used a quantitative research methodology. The total sample was composed by 367 teachers. The results shows a high leveles of dissatisfaction and low motivation by teachers in Benguela's research context.
\end{abstract}

Keywords: Motivation, teacher satisfaction, professional development, secondary education

\section{Resumen}

Son pocas las investigaciones sobre el tema de la motivación y satisfacción en el trabajo, aunque si existen algunos estudios sobre la motivación de los docentes y la satisfacción en su ámbito laboral, pero en Angola emergen estos aspectos como nuevos en el campo de la investigación científica y más concretamente dentro del campo de la organización escolar 
y la gestión, entendiendo éste como un conjunto de conocimientos y acciones entre las personas que confluyen en valores y prácticas compartidas que se manifiesta a través del trabajo. El presente trabajo tiene por objetivo estudiar la motivación y satisfacción del profesorado para el desempeño de su profesión en Benguela (Angola). A través de ella tratamos de describir cómo la motivación y la satisfacción pueden influir en el desarrollo profesional de los docentes que trabajan en Educación Secundaria en Benguela. Para ello se empleó una metodología de investigación de corte cuantitativa. La muestra estuvo compuesta por un total de 367 profesores. Los resultados muestran altos niveles de insatisfacción laboral y una baja motivación por parte de los profesores en el contexto de Benguela.

Palabras clave: motivación, satisfacción del profesorado, desempeño profesional y educación secundaria.

\section{Introducción}

Este trabajo científico tiene una relevancia con respecto al modelo de la motivación y la satisfacción de los profesores, ayudándoles a mejorar sus acciones didácticas educativas y motivarse para hacerlo. La motivación es un refuerzo de cualquier actividad laboral que busca resultados tangibles.

La búsqueda de las razones relativas a la cuestión de la motivación y la satisfacción de los profesores es reducida, aunque ya hay algunos estudios sobre la motivación de los docentes y la satisfacción en el mundo, pero en la realidad de Angola emerge como nueva en el campo de la investigación científica. Este tema, como hemos mencionado en el resumen, se integra dentro del ámbito de la organización y gestión de instituciones educativas.

Se sabe que los primeros pasos de la investigación sobre la satisfacción laboral docente se inició en los años 70, las relaciones entre la satisfacción laboral y la calidad de vida, la salud mental y el entorno familiar (Seco, 2000, p. 37). Estos elementos están estrechamente relacionados entre sí, con el fin de fomentar un comportamiento deseable en el desempeño de actividades profesionales de cualquier maestro individual y especialmente la región de Benguela.

Resaltar que las escuelas necesitan maestros altamente motivados y satisfechos con el desempeño de sus actividades, hasta el punto de crear entre ellos un ambiente armonioso y agradable que luego se traduce en satisfacción en el trabajo que puede ayudar en el buen desempeño de los mismos y los "frutos" deseados. 


\section{La motivación y satisfacción laboral como aspectos clave en el desempeño de la labor docente}

La motivación es un factor muy importante en la realización de cualquier actividad del hombre, tanto profesional como social. Es una gran fuerza que lleva al individuo a crear, como todo lo que hace y lo hace con la perfección $\mathrm{y}$ el amor. La motivación para el trabajo trae varias consecuencias psicológicas positivas, como la sensación competente y útil y mantener nuestra autoestima (Ardila, 1986 y Murray, 1967, p. 80). La motivación y la satisfacción de los profesores se observa en los recursos educativos que utilizan en su práctica profesional diaria.

La elección de este tema se justifica por el hecho de que se encontró deficiencias en la motivación y la satisfacción de los profesores de estas escuelas, lo que significa que sólo funcionan para el cumplimiento del programa, para la subsistencia y no para su logro profesional.

La importancia de este tema, se consolida en el hecho de que los profesores poseen un saber profesional único y esta cualidad hace que su propio razonamiento sobre la actividad profesional que realizan y su tarea docente educativa no se puede desligar de su función social que le permite tomar sus propias decisiones de manera consciente y racional por sus conocimientos y poderlas utilizar para facilitar el aprendizaje a los estudiantes.

El profesor tiene una peculiaridad que no se puede separar de su papel como constructor social como alguien que tiene la responsabilidad de construir social y humanamente la apertura de nuevos horizontes para el bien común en la educación en la multiplicidad y la interactividad de sus dimensiones: cognitivas, afectivas, psicomotoras, lingüísticas, de relación, comunicación y ética.

Anteriormente el papel del profesor fue el único asociado con la función de inculcar e impartir conocimientos a los estudiantes. Actualmente parece que el papel del profesor es más amplio y asume el papel de guiar a los estudiantes de para que construyan el conocimiento por sí mismos, asegurando así una mejor formación de la personalidad, capaz de satisfacer las demandas de la sociedad y por lo tanto se convierten en personas competentes en el ejercicio de sus funciones.

Para que el profesor cumpla dicha función, debe estar muy motivado y satisfecho profesionalmente y transferir esta motivación a sus alumnos para crear un ambiente de armonía en la escuela. En este contexto, es necesario que los gestores de la escuela "directores de la escuela" puede mantener la satisfacción motivando permanente a sus empleados, usando técnicas para ello, en el sentido de mantener el ritmo normal de su trabajo.

El saber profesional específico de un profesor no se puede entender si separamos la función social de los docentes como a alguien que la sociedad 
le ha encomendado o confiado la tarea de crear contextos de desarrollo humano que involucren al estudiante en multiplicidad e interactividad de sus dimensiones cognitivas, afectivas, psicomotrices, lingüísticas, de relación, comunicación y ética (Roldão, 1998b, Pág. 5). Debe haber una conexión entre el plan de estudios que es la esencia de la escuela, y el maestro que es el profesional que trabaja en el mismo plan de estudios conjunto de aprendizaje que se espera que aprender en la escuela, teniendo en cuenta el contexto histórico y social de la sociedad en que se desarrolla (Roldán, 1999, p. 47).

El estar satisfechos profesionalmente es una gran ventaja tanto para el profesor como para los estudiantes y en general para toda la institución escolar y en la sociedad en la que esta inserta. La satisfacción en el trabajo del maestro se traduce en una gran ganancia en el proceso de aprendizaje, la producción de estos resultados son muy positivos, por lo que puede beneficiar al desarrollo de la sociedad de Benguela y más allá.

La motivación y la satisfacción es algo altamente involuntario, y todo depende de cómo el individuo se siente en el lugar de trabajo con sus colegas, estudiantes y la dirección de la escuela. Sin embargo, si el clima laboral es bueno y hay una relación interpersonal agradable entonces no necesita mucho esfuerzo para mantener equilibrado, dispuesto y motivado al equipo a cumplir con el desempeño de sus actividades y alcanzar todos los objetivos comunes de la institución. Se cuenta mucho con la dinámica del responsable de la institución para mantener la química o el buen ambiente en el lugar de trabajo, consiguiendo que cada uno este allí para sentirse cómodo como si estuviera en familia. La motivación es involuntaria, ya que no tiene que ser premeditada, además de los propios acontecimientos se mantendrá o aumentará o disminuirá el estado emocional de los profesionales, por lo tanto, la motivación causa la satisfacción o desmotivación causando de esta manera la insatisfacción laboral.

Transformar el lugar de trabajo en un ambiente agradable y cómodo promueve una sensación de bienestar nos afecta a todos (Bruce A, 2006, p.14). El profesor debe sentirse bien en el lugar de trabajo y tener la institución en la que enseña, como su segundo hogar, con la libertad de expresión, así como la autonomía de contribuir para el desarrollo del trabajo con el fin de alcanzar los objetivos comunes propuestos por la escuela.

Sin embargo, la satisfacción laboral tiene que ver con las motivaciones intrínsecas del individuo en relación con la ejecución del trabajo (que lo hace) y el medio ambiente de trabajo (dónde trabajan) y lo que significa este trabajo para él (Tonet Helena, Ana M. V Reis, Luiz CB Jr. y EB aria Costa, 2007, p. 146). 
La motivación en el trabajo debe partir del interior de la persona que la ejerce, porque nadie puede motivar a nadie si no tiene el interés inicial y el trabajo debe tener un significado único en la vida del profesional. La motivación no se enseña sino que se contamina con los buenos actos de convivencia. Así que el director de la escuela siempre debe ser ejemplar en todo, que demuestre con acciones prácticas y no sólo guiar y criticar. Más bien debe ser el que de vez en cuando lance elogios por el buen trabajo realizado por el equipo.

La gestión del capital humano es lo más complejo y lo más importante dentro de una organización, por lo que debe hacerse con cautela, y procurar entender las motivaciones de cada uno en el día a día, semanalmente y mensualmente, ya que cada persona trae sus problemas individuales y no se entienden asociados en el trabajo profesional. En esto, la retroalimentación negativa debe estar libre de la convivencia en el trabajo.

Vale la pena señalar la relación existente entre la motivación y satisfacción laboral, ya que ambos trabajan en el sistema y deben ir de la mano porque uno complementa al otro, y todo profesional motivado realiza mejor y con más exactitud sus actividades y procura la satisfacción tanto a nivel personal, profesional, como socialmente.

\section{Método}

Como ya se mencionó anteriormente, este estudio tiene como finalidad realizar un diagnóstico sobre la motivación y la satisfacción de los docentes para el desempeño profesional en las escuelas del 1er ciclo de la educación secundaria en la ciudad de Benguela (Angola).

En este contexto, los aspectos didácticos y metodológicos que han guiado el trabajo de investigación y los progresos realizados, se definen en los siguientes objetivos.

\section{Objetivos}

El Objetivo General de esta investigación es "Analizar las particularidades de la motivación y la satisfacción laboral de los profesores de las escuelas urbanas públicas del primer ciclo de la Educación Secundaria en la ciudad de Benguela (Angola)".Para ello seguimos los siguientes Objetivos Específicos:

- Describir las características de la motivación y la satisfacción de los docentes para el desempeño profesional en las escuelas de primer ciclo de educación secundaria en la ciudad de Benguela (Angola).

- Definir la política que puede ayudar a mejorar la motivación y la satisfacción de los docentes para el desempeño profesional en las escuelas de primer ciclo de educación secundaria en la ciudad de Benguela (Angola). 
- Analizar la relación entre la motivación, la satisfacción y el desempeño profesional de los docentes en las escuelas de primer ciclo de educación secundaria en la ciudad de Benguela (Angola).

\section{Muestra}

La muestra de este estudio ha estado conformada por 367 profesores que respondieron a una encuesta que se le aplico, siendo 211 mujeres y 156 hombres.

La población del estudio representa un $50 \%$ de los 735 profesores que constituyen la población total del objetivo. Obsérvese el siguiente cuadro con los participantes de esta investigación:

\begin{tabular}{|c|c|c|c|c|}
\hline $\begin{array}{c}\text { Zonas } \\
\text { Escolares }\end{array}$ & Escuela & Participantes & Masculino & Femenino \\
\hline $\mathbf{A}$ & 10 de Febrero & 169 & 71 & 98 \\
\hline $\mathbf{D}$ & Tomás Ferreira & 94 & 46 & 48 \\
\hline $\mathbf{E}$ & 22 de Noviembre & 104 & 39 & 65 \\
\hline & Total & 367 & 156 & 211 \\
\hline
\end{tabular}

Tabla 1: población

\section{Instrumentos}

Los instrumentos de investigación seleccionados y aplicados fueron la entrevista y el cuestionario en encuesta. De acuerdo con Marconi y Lakatos (2006, pp. 92-97), la entrevista es un encuentro entre dos personas para obtener una información sobre un determinado tema, en una conversación de carácter profesional. Es el procedimiento utilizado en la investigación social, ayuda en la recogida de datos, en el diagnóstico y tratamiento de un problema social. La encuesta, según Ghiglione y Matalon (2005, p. 7), es un cuestionamiento acerca de una situación particular, que abarca a los individuos, con el fin de generalizar. Es, por lo tanto, obtener un conjunto de puntos de vista, la interpretación de ellos y generalizarlos. Los mismos autores añaden que los problemas teóricos y metodológicos que plantea la práctica y el uso de la investigación vinculados a las características de este método. El cuestionario es un instrumento de recolección de información que consiste en una serie de preguntas ordenadas que debe ser respondida por escrito y sin la presencia del entrevistador. Para este trabajo se había agotado el tipo de entrevista semiestructurada que de acuerdo a Fick, Uwe (2005, p 94) busca incorporar preguntas más o menos abiertas en el guión. Con ello se espera que los encuestados den respuesta de forma clara y libremente a estas preguntas. 


\section{Diseño metodológico y procedimiento}

En la ejecución de esta investigación se utilizaron los métodos y procedimientos metodológicos que se han referido. De acuerdo con su naturaleza, esta investigación se centra en una forma de investigación cualitativa, que pretende generalizar la fiabilidad y validez del estudio a través del procesamiento de datos cuantitativos de los procedimientos informáticos (Ediciones Universidad de Salamanca, 2007, p. 29). Elegimos un enfoque descriptivo, que según Gonçalves, VG (2005, p. 8) en su metodología de investigación educativa, afirma que "la investigación descriptiva refleja las características observables y generales que tienen por objeto aclarar, clasificar, establecer las propiedades más importantes de las personas, grupos, comunidades o cualquier otro fenómeno que se somete a análisis".

En vista de Zassala (2013, pp. 42-43), "la investigación descriptiva es la investigación que busca determinar la naturaleza y el alcance de las condiciones existentes. Este método tiene la única finalidad es describir las condiciones existentes o fenómenos que se producen ".

Defiende una lógica inductiva en el proceso de investigación, donde los datos son recogidos en función de los supuestos definidos (hipótesis) que tienen que ser puestos a prueba, pero más con el objetivo de partida de los datos que se encuentran en ellos y regularidades que subyacen a las generalizaciones que son cada vez más amplios.

En el enfoque inductivo nada se establece de inmediato, sino que deben ser obtenidos a partir de las experiencias personales de los participantes.

Esta metodología tiene como objetivo hacer que los resultados de este proyecto de investigación pueden traducirse en cambios y ajustes en el punto de la mejora de la motivación y la satisfacción de los profesores para el buen desempeño profesional en las escuelas de I ciclo en busca de la eficiencia del proceso de la enseñanza educativa.

\section{Resultados}

En este apartado, nos proponemos presentar y describir brevemente y con claridad los resultados de la investigación realizada a los profesores de las escuelas de I ciclo de educación secundaria en Benguela (Angola). 


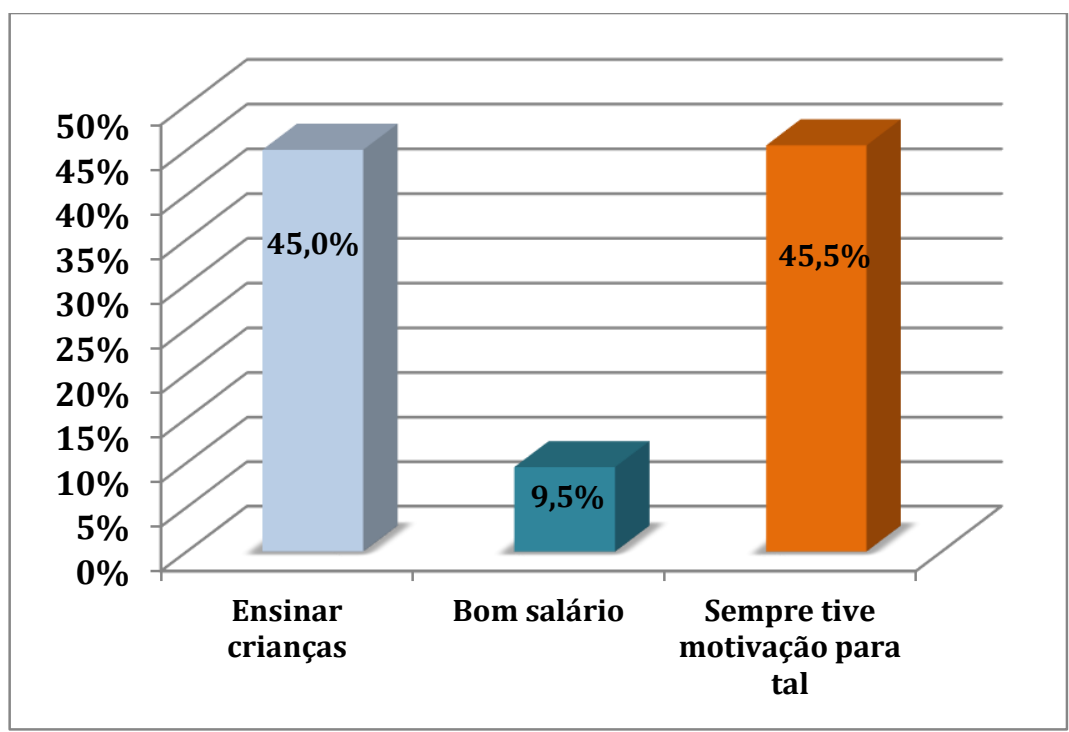

Gráfica 1: Motivaciones para ser profesor

Vista la pregunta ¿Qué te motivó que ser profesor/a?, el $45.5 \%$ dijo que siempre tuvieron la motivación por la enseñanza, mientras que el 9,5\% se han unido al Ministerio con un sueldo bueno teniendo un buen nivel académico, y el $45 \%$ respondió que enseñar a los alumnos es lo que le motivó para ser profesor.

Teniendo en cuenta los resultados obtenidos de la muestra se puede percibir que la mayoría de los profesores han tenido una vocación por la profesión docente. Por lo tanto, creemos tener resultados satisfactorios en este sentido, dado que los indicadores apuntan a una satisfacción intrínseca de los docentes a la profesión. Para que el proceso de gestión fluya, los gestores deben asumir con responsabilidad y rigor su tarea.

Por otro lado, es necesario que los directores, gestores e instituciones escolares acrediten los esfuerzos para aplicar una serie de estrategias para elevar el nivel de motivación de los profesores en el proceso de enseñanza para que puedan manifestarse en el desempeño de los mismos. 


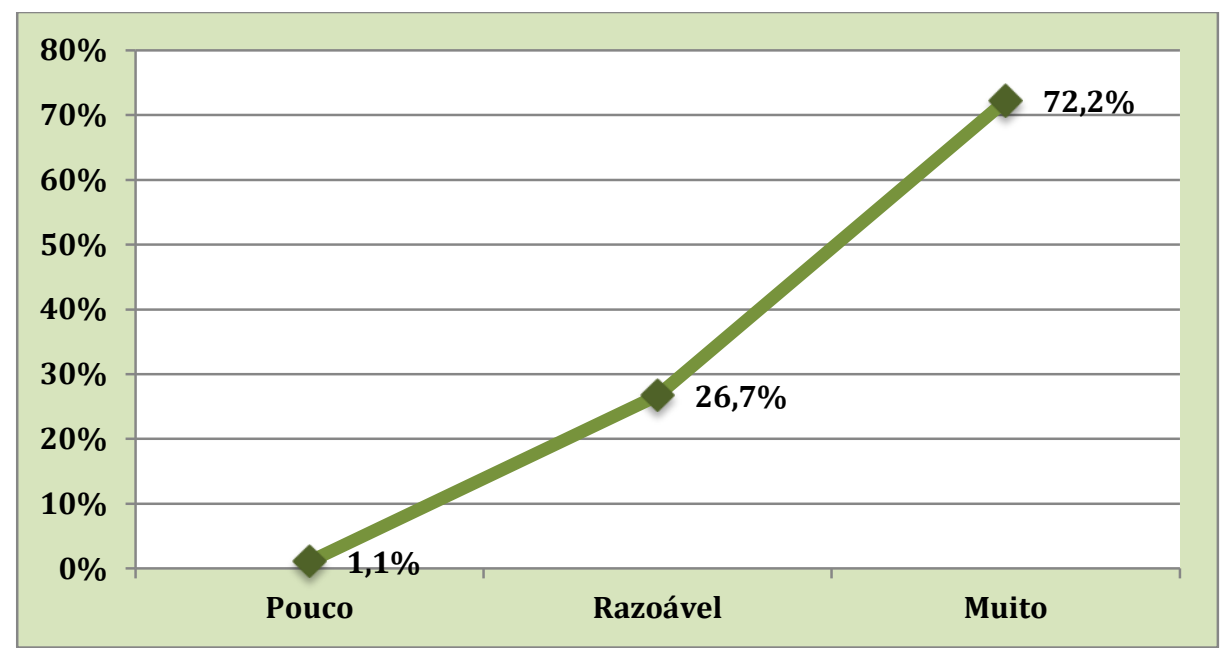

Gráfica 2: Opinión sobre si les gusta ser profesor

De la cuestión ¿le gusta ser un maestro?, más de la mitad de los encuestados respondieron que les gusta muchísimo ser maestros. Teniendo en cuenta estos resultados se puede ver que a pesar de tener un mayor número de profesores que manifiestan positivamente para ser maestros, todavía se puede decir que los resultados no son tan satisfactorios para la profesión ejercida por los maestros. Dados los resultados, se observa que existe una insatisfacción entre los maestros y que a menudo esta provocada por problemas para poder realizar sus actividades.

Por lo tanto, es necesario establecer los mecanismos adecuados para facilitar y contribuir al proceso de gestión.

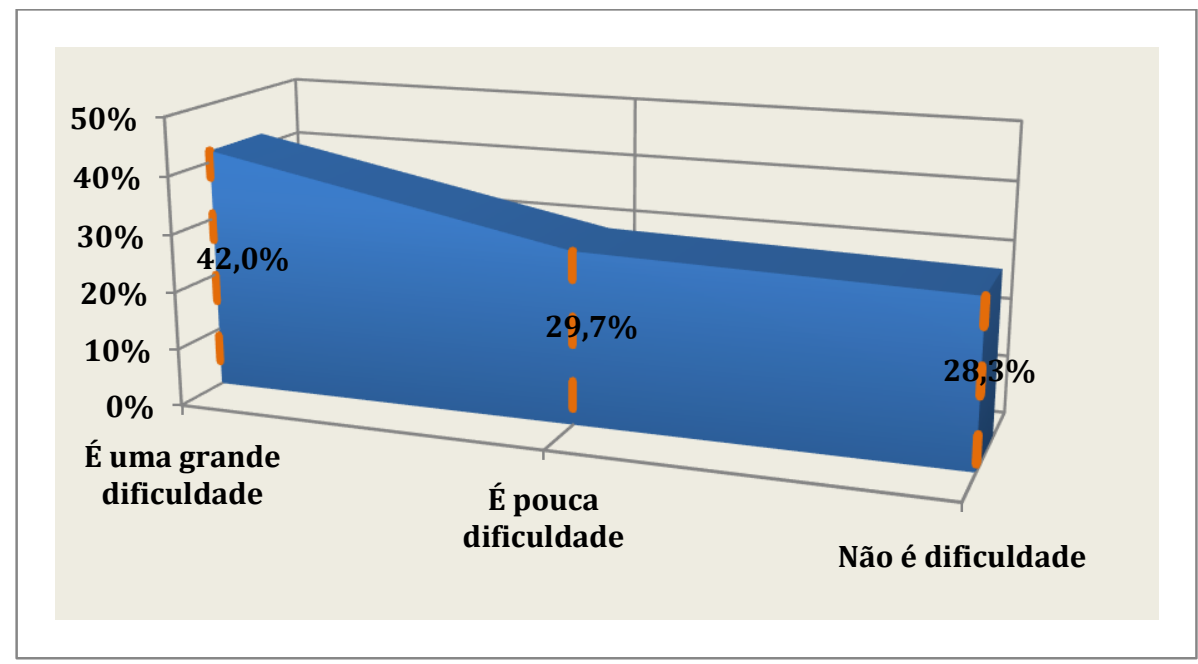

Gráfica 3: Relación con las familias de los estudiantes 
En cuanto a la cuestión sobre la relación con las familias de los alumnos, aquí tenemos un gran "talón de Aquiles", ya que mirando el gráfico esta muy claro que la mayoría y cerca del $70 \%$ de los profesores afirman tener dificultades en la relación con las familias de los estudiantes y que los familiares no se preocupan mucho por el intercambio entre ellos y la escuela con el fin de entender cómo es el proceso de enseñanza - aprendizaje de sus hijos y esto ha sido un gran problema para los maestros porque no han sabido resolver determinadas situaciones que se producen durante el proceso que se desarrolla con los estudiantes. Frente a esta realidad hay que tomar la iniciativa.

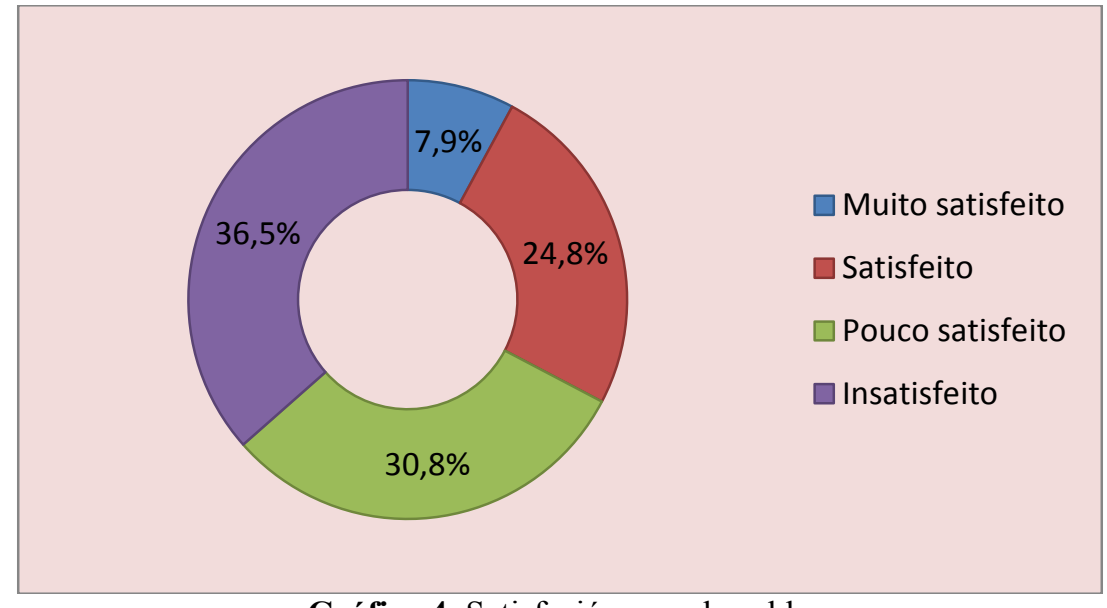

Gráfica 4: Satisfación con el sueldo

La pregunta aplicada: ¿Está satisfecho con su sueldo?, el 36,5\% dice que no están satisfechos y el 30,8\% dicen que están poco satisfechos. Haciendo un análisis global, se aprecia insatisfacción por encima de la media, mientras otros dicen estar ligeramente satisfechos.

Los resultados obtenidos en la muestra indican que los maestros se sienten insatisfechos sobre todo en relación con el salario, lo que sin duda ha afectado no sólo el progreso del proceso de enseñanza educativa, así como su propio rendimiento.

El proceso de gestión del capital humano sólo tendrá éxito, si en serio se miran estos aspectos para asegurar las condiciones mínimas para mejorar el buen desempeño docente. Por lo tanto, estamos de acuerdo con Herzberg citado por Teixeira (2005), en su teoría de las necesidades cuando afirma que los factores de higiene incluyen los salarios y que son capaces de reducir o aumentar la insatisfacción, pero no pueden dar lugar a la motivación. (Tan, S; 2005, pp. 147-148). 


\begin{tabular}{|c|l|l|l|l|}
\hline & Freqûencia & Percentagem & $\begin{array}{l}\text { Percentagem } \\
\text { válida }\end{array}$ & $\begin{array}{l}\text { Percentagem } \\
\text { acumulada }\end{array}$ \\
\hline $\begin{array}{c}\text { É uma grande } \\
\text { dificuldade }\end{array}$ & 187 & $\mathbf{5 1 , 0}$ & 51,0 & 51,0 \\
\hline $\begin{array}{c}\text { É pouca } \\
\text { dificuldade } \\
\text { Não é } \\
\text { dificuldade } \\
\text { Total }\end{array}$ & 85 & 25,9 & 25,9 & 76,8 \\
\hline
\end{tabular}

Tabla 2: Tabla de frecuencias y porcentajes. Número excesivo de alunos por turno.

En cuanto a la cuestión si hay un excesivo número de alumnos por clase; más del $50 \%$ de la muestra fueron unánimes en señalar qué es un grado de dificultad el excesivo número de alumnos por clase, lo que obstaculiza gravemente el desarrollo del proceso de enseñanza-aprendizaje. Estos planteamientos se nos dice en las palabras de (Libâneo: 366), sin embargo, es necesario cumplir con las condiciones que tiene la organización y el buen desarrollo de la enseñanza para que el profesor pueda dirigir la clase a la perfección y tener una escuela con calidad, respetando al mismo tiempo los principios de la gestión escolar.

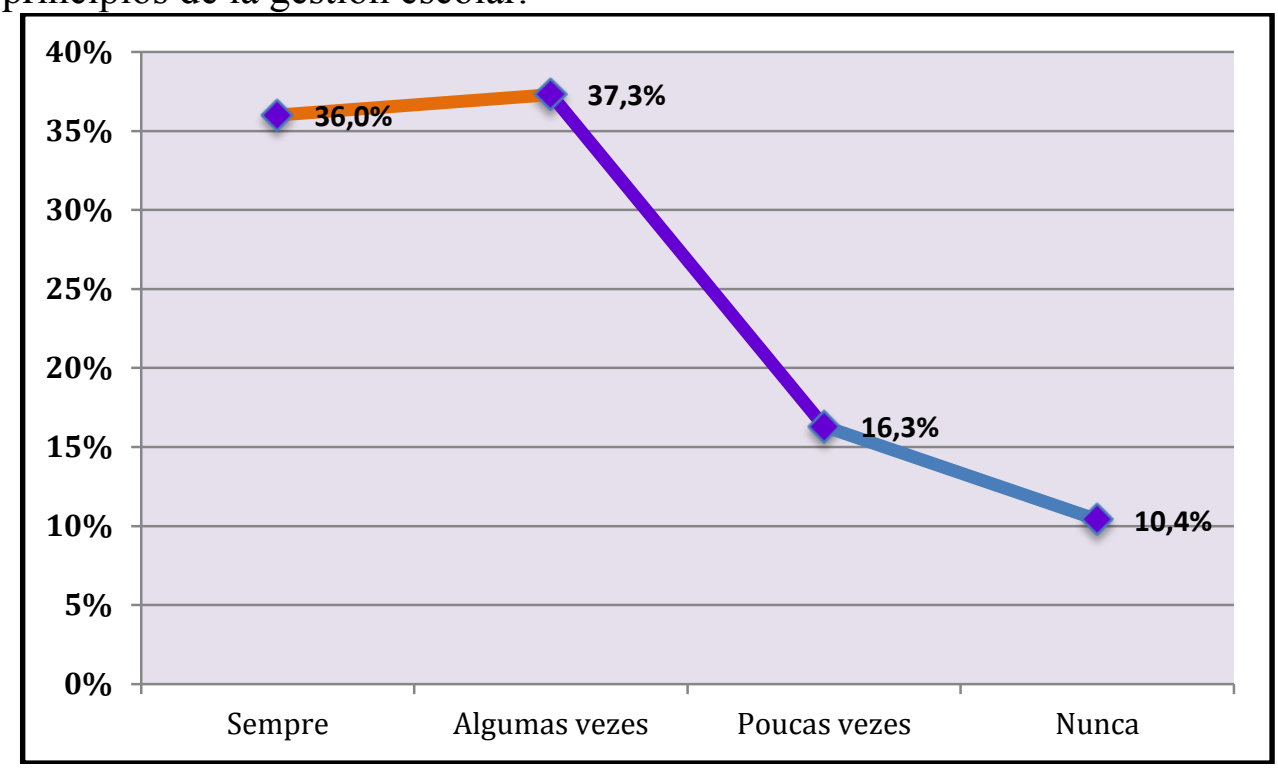

Gráfico 5: Formación en su centro de cursos de actualización docente.

En la pregunta: ¿En su escuela se ha llevado a cabo la formación de seminario de actualización en su capacitación?, los aspectos más destacados respondidos es que el seminario se realiza con poca frecuencia y la actualización es una formación continua del profesorado. 
En cuanto a los resultados de la muestra se puede inferir que la mayoría de los profesores no siempre declaran realizar el seminario de formación y actualización en sus escuelas, dicho fallo hace que el profesor no adquiere nuevos conocimientos de forma continua o desarrollar sus habilidades para el ejercicio de sus acciones. Si el profesor no tiene la capacitación continúa ¿cómo va a superar sus fracasos?. Es necesario que haya intercambio de conocimiento y actualización constante en la práctica de la enseñanza educativa se deriva la necesidad que los gerentes de las escuelas se esfuercen para facilitar la formación continua de los profesores para que desarrollen habilidades que les lleva a mejorar su conocimiento didáctico-profesional, para ofrecer alternativas de trabajo dirigidas a alcanzar mejores resultados.

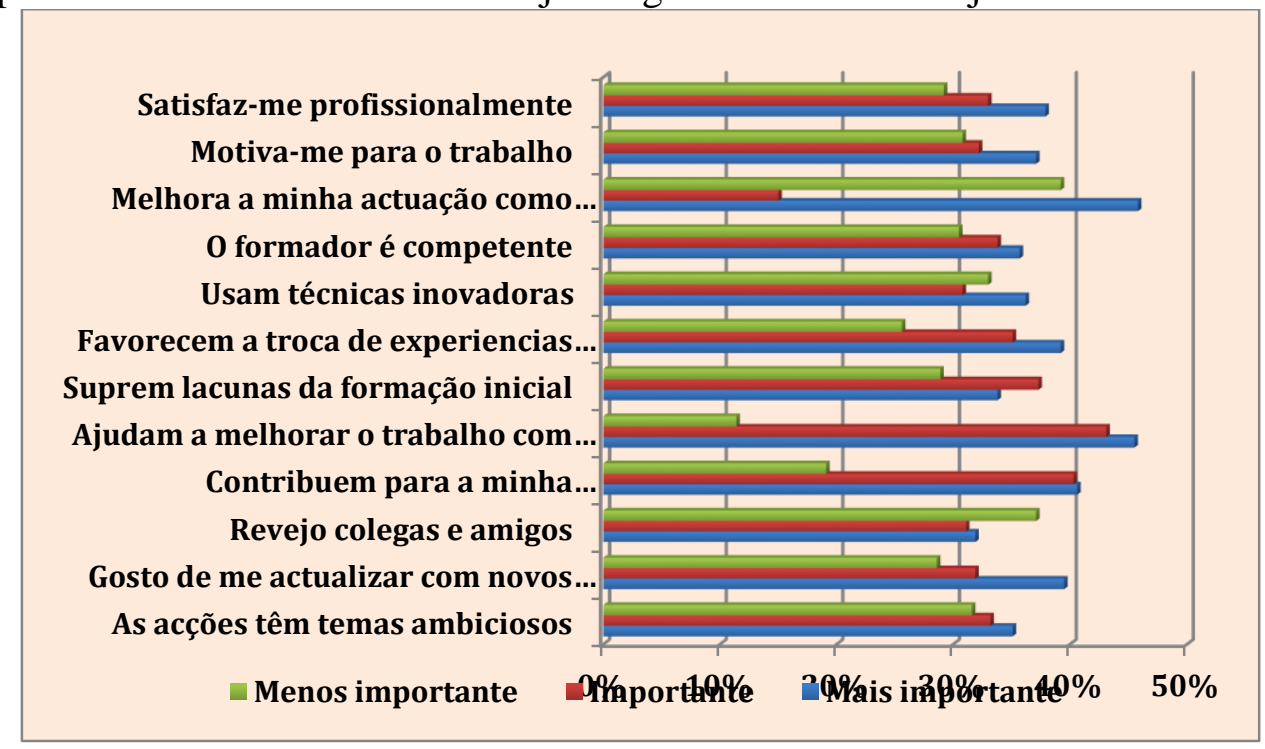

Gráfico 6: Frecuencia de asistencia a cursos de formación.

En cuanto a la pregunta de por qué asistir a un curso de formación, los indicadores apuntan principalmente los siguientes elementos motivadores: porque me satisface profesionalmente, me motiva a trabajar, mejorar mi desempeño como profesor, ayudar a mejorar el trabajo con los estudiantes y me gusta actualizarme con los nuevos conocimientos.

Los factores aquí mencionados indican el perfil del verdadero profesor y su madurez profesional que se materializa en un conjunto de habilidades de las tareas realizadas por él. Es una mente sana, dispuesta a buscar nuevos horizontes de conocimiento que tienen como objetivo proporcionar una calidad en su carrera profesional. 


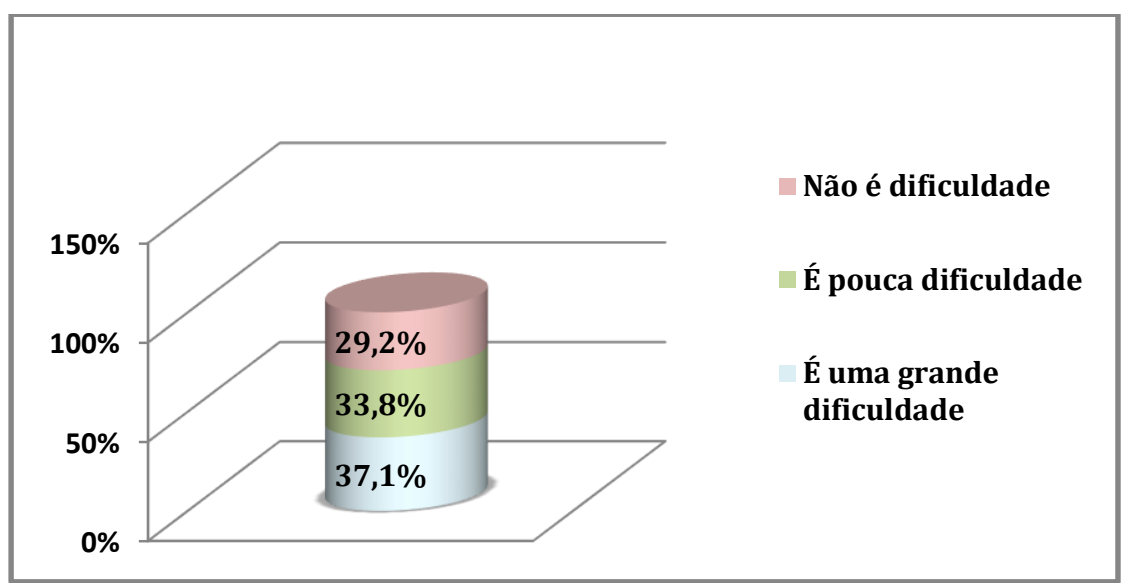

Gráfica 7: Tiempo dedicado a la correction de las pruebas de evaluación.

De acuerdo a la pregunta que indica el tiempo requerido para la corrección de las evaluaciones, el 37,1\% refiere que es una gran dificultad esto porque a menudo el calendario de las pruebas y los resultados de visualización preparado por el Ministerio de Educación no es flexible para permitir a la dirección de las escuelas los días para la corrección de las evaluaciones. Sin embargo, es necesario que los profesores desarrollen competencias pedagógicas que ayuden a interactuar permanentemente con el contexto vivenciado y estudiarlo con el fin de buscar métodos de trabajo sistemáticos para dar respuestas rápidas y sabias en el sistema educativo. Blanco, define la competencia como resultado de la interacción del individuo con el medio ambiente que lo rodea, como resultado de un aprendizaje o adaptación (Neves, A. L., 2002. P. 41).

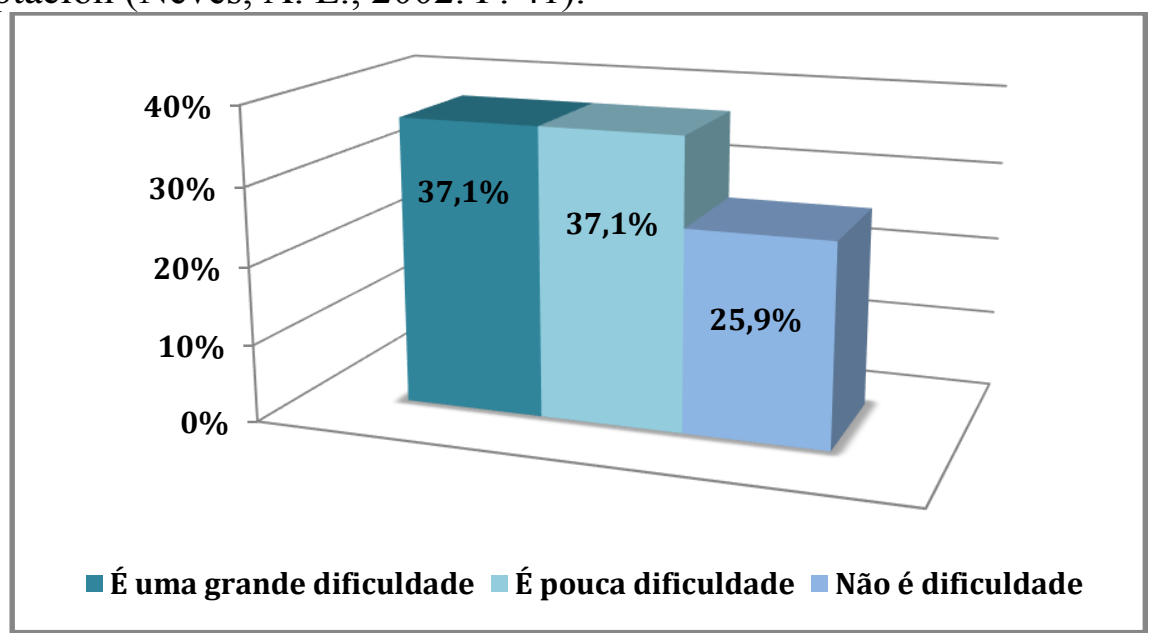

Gráfica 8: Clarificación de tareas a realizar.

La pregunta dirigida a los encuestados respecto a la falta de claridad en lo que tienen que hacer, se ve aquí igual, que el $37,1 \%$ de las respuestas lo 
ven tanto como una gran dificultad, como con poca dificultad. En el análisis de las respuestas podemos entender que hay una preocupación en la mayoría de los profesores sobre este tema. Desde nuestro punto de vista, es difícil de secuenciar el comportamiento de las acciones del profesor si no hay claridad en los objetivos tangibles, por lo tanto tiene que haber unas orientaciones que pueden impulsar al profesorado para llevar a cabo su trabajo.

Creemos que a menudo se lleva a cabo la formación de liderazgo para capacitar a los directores de escuela.

\begin{tabular}{|c|l|l|l|l|}
\hline & Freqûencia & Percentagem & $\begin{array}{l}\text { Percentagem } \\
\text { válida }\end{array}$ & $\begin{array}{l}\text { Percentagem } \\
\text { acumulada }\end{array}$ \\
\hline $\begin{array}{c}\text { É uma grande } \\
\text { dificuldade }\end{array}$ & 153 & $\mathbf{4 1 , 7}$ & 41,7 & 41,7 \\
É pouca dificuldade & 121 & 33,0 & 33,0 & 74,7 \\
Não é dificuldade & 93 & 25,3 & 25,3 & 100,0 \\
Total & 367 & 100,0 & 100,0 & \\
\hline
\end{tabular}

Tabla 3: Tabla de frecuencias y porcentajes. Condiciones de trabajo

En cuanto a la cuestión expuesta en la tabla de frecuencias anterior, el $41,7 \%$ de los encuestados declaran tener una gran dificultad en las condiciones de trabajo ofrecidas por las escuelas. No queremos señalar grandes condiciones, pero lo más básico son las aulas de las escuelas que todavía existen estudiantes en los balcones, los materiales didácticos, las condiciones de carteras, pizarra, como la tiza ha causado muchas complicaciones en la salud de los maestros y no sólo, los laboratorios y las bibliotecas escolares para facilitar la investigación de los profesores y estudiantes, porque no podemos hablar de profesor de investigación si no las condiciones para tal fin establecida. Para tener un buen resultado en la educación de los ciudadanos es totalmente necesario mejorar las condiciones de trabajo en las escuelas públicas. Por lo tanto estamos de acuerdo con Herzberg, Mausner y Snyderman (1993), cuando consideran que el trabajo juega una importancia insustituible en la vida del hombre, es un tema candente y actual que ha despertado la atención de todos los trabajadores con el fin de hacernos reflexionar en sus prácticas diarias que causan satisfacción a unos y a otros la insatisfacción y que de cualquiera de las maneras, dañará el desempeño del trabajador. 


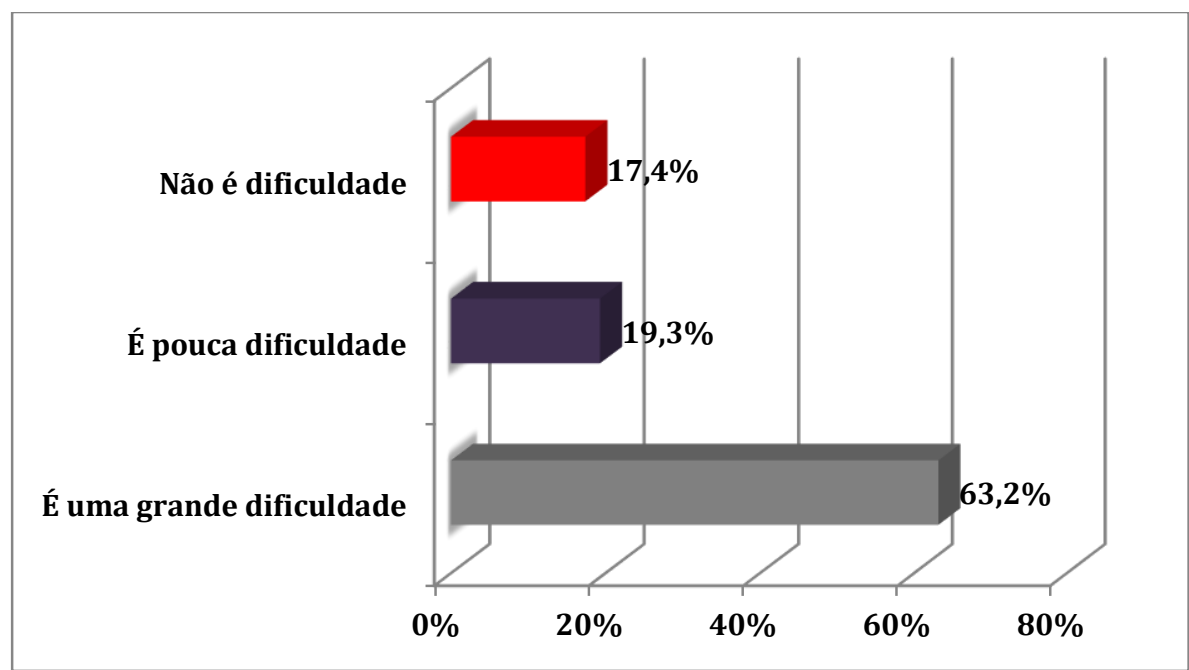

Gráfica 9: Falta de salario adecuado.

Más de la mitad de los profesores encuestados que corresponden a un $63,2 \%$, afirman que la falta de un salario adecuado es una de sus principales dificultades. Si recordamos las teorías de las necesidades de diferentes autores vemos que el salario es una de las necesidades más consideradas, que puede influir en gran medida en las necesidades más altas del trabajador. No podemos ver la situación salarial como un mero problema. Es preciso que el gobierno se preocupe por el estado de motivación y el grado de satisfacción de sus empleados directos (funcionarios) y siempre debe estar atento a las causas de desmotivación de los trabajadores en todos los sectores para que haya una buena colaboración en el logro los objetivos declarados, porque la satisfacción es señal de rendimiento, o sea, no hay un rendimiento eficiente en el trabajo si no es satisfactorio lo que hacemos.

\section{Conclusiones}

Después de una larga etapa de investigación, hemos llegado a la conclusión de que existe un grado de motivación e insatisfacción dentro de las escuelas de profesionales de la educación de las escuelas de I ciclo de enseñanza secundaria en Benguela (Angola), teniendo en cuenta las siguientes insuficiencias encontradas:

- Falta de un marco salarial, insuficiente aplicabilidad de seminarios en las escuelas, la falta de claridad sobre lo que se hará y el excesivo número de estudiantes por clase son un obstáculo importante en la motivación y la satisfacción en la mayoría de los profesores de las escuelas de I ciclo en la ciudad Benguela.

- Cómo estrategias de mejora de la motivación y satisfacción para el desempeño de actividades, no es más que velar por el reajuste salarial 
de los profesores una vez que la mayoría han completado un grado, pero ostentan por un salario inferior de técnico medio y después a los 5 años pueden estar expuestos a ascender a otra categoría. Los seminarios son frecuentes y sistemáticos en las escuelas o nivel de Zips (Zonas Influencias pedagógicos), no sólo para los profesores con el fin de interactuar e intercambiar experiencias, sino también para los gestores de la escuela de forma que los capacite cada vez más y pueden orientar con claridad a sus empleados. El excesivo número de alumnos por clase, debe ser pautado a 35 estudiantes por clase para dar al profesor la posibilidad de conocer las características individuales de cada estudiante y que pueda ayudar a superar sus dificultades.

- En cuanto a la relación triple entre motivación, satisfacción y desempeño o rendimiento, de acuerdo con la idea de Machado y Soares (2014), que la "satisfacción en el trabajo es un constructo que es una respuesta a un estado emocional positivo o una actitud positiva hacia el trabajo y la experiencia en el lugar de trabajo, esta satisfacción se presenta como sentimiento y forma de ser positiva, dada la profesión y sentimientos contrarios a materializarse-la presencia de insatisfacción "(Machado, M., Smith, V en absoluto. 2014, p 50), confirmó que existe una relación importante entre la motivación, la satisfacción y el desempeño profesional ya que la motivación es la fuerza que impulsa al individuo a realizar cualquier actividad que busca resultados tangibles, es un elemento clave para elevar el grado de satisfacción por lo que haces y juntos son una gran palanca para el desempeño profesional de los profesores en las escuelas del I ciclo en Benguela. La satisfacción es señal de rendimiento, desempeño, porque todo profesional satisfecho es mejor, se siente mejor.

\section{References:}

1. Bruce, A. (2006). Como Motivar Sua Equipe. Editora Sextante. Rio de Janeiro (Brasil).

2. Ediciones Universidad Salamanca, (2007). Enseñanza anuario interuniversitario de didáctica. Edicione Universidad Salamanca.

3. Fick, U. (2005). Métodos qualitativos na investigação científica. Editora: Monitor- projectos e Edições, Lda.

4. Gonçalves, V. G. (2005). Metodologia de investigação educativa, Ed. ISCED/Benguela (Angola).

5. HerzBerg, F., Mausner, B., \& Snyderman, B. (1993). The Motivation to Work. New Brunswick and London: Transaction Publishers. 
6. Libâneo, J.C; Oliveira, J.F; Toschi, M. Seabra. (2008). Estrutura e organização da educação Brasileira. Cortez editora, $6^{a}$ edição.

7. Machado, M. de L., Virgílio, M. S. at all., (2014). Satisfação e Motivação dos Académicos no Ensino Superior Português. Edições de Almedina. Coimbra (Portugal).

8. Marconi, M. de A., e Lakatos, E. M. (2006). Tecnicas de pesquisa, $6^{a}$ edição, Editora atlas. São Paulo (Brasil).

9. Neves, A. L. (2002). Motivação para o trabalho. 2a Edição, EditoraRH. Lisboa (Portigal).

10. Roldão, M. C. (1998). Os desafios da profissionalidade docente e o currículo. Aveiro CIFCP da Universidade de Aveiro.

11. Roldão, M. C. (1999). Os professores e a gestão do currículo. Porto Editora.

12. Teixeira, S. (2005). Gestão das organizações. Verlag Dashofer, $2^{\mathrm{a}}$ edição.

13. Zassala, C. (2013). Iniciação à pesquisa científica, Mayamba Kunhyonga. Luanda/Angola, $2^{\text {a }}$ edição. 